\title{
Mathematical Models for Flow of Chyme during Gastrointestinal Endoscopy
}

\author{
Ranadhir Roy, Fabiola Rios, Daniel N. Riahi \\ Department of Mathematics, University of Texas-Pan American, Edinburg, USA \\ E-mail: rroy@utpa.edu \\ Received March 11, 2011; revised March 25, 2011; accepted March 29, 2011
}

\begin{abstract}
Intestinal infection has become a common disease in human and endoscopy can be a powerful means in diagnosis of intestinal illnesses. Mathematical models are developed for an inserted endoscope on the flow of chyme in the small intestine considering a Newtonian incompressible fluid flow, under an axisymmetric condition, in a cylindrical annulus between the small intestine and the endoscope. We obtain novel mathematical expressions for the pressure drop, forces exerted by the endoscope on the flow of chyme, and the force exerted by the chyme on the intestine for one wave length of the peristaltic rush wave. We also investigate and calculate the flow velocity and pressure for different flow rates and the wave lengths. The results are presented, and discussed for the cases and conditions under which pressure, pressure drop can be positive or negative and the forces can be acted either by the intestine or endoscope on the flow or vice-versa.
\end{abstract}

Keywords: Mathematical Models, Gastrointestinal, Chyme Flow, Endoscopy, Flow Modeling, Newtonian Incompressible Fluid Flow

\section{Introduction}

Intestinal infection has become a rather common disease in living systems such as that of human. This illness is often caused by the environmental pollution like water pollution that can lead to constipation, distention, etc. Due to such infectious conditions, a strong wave called peristaltic rush develops and it travels relatively long distances in a few minutes in small intestine, which is a convoluted tube of about 6-7 $\mathrm{m}$ in length and average radius about $1.25 \mathrm{~cm}$ lying in the central and lower parts of abdomen. The functions of the small intestine in human, which is called gastrointestinal tract, are for digestion and absorption. It is known that endoscopy can be a powerful means in diagnosis and management of various types of intestinal illnesses.

Although there have been studies about transport of chyme flow by the peristaltic rush wave [1-6], but relatively little is known about the adverse effect of an inserted endoscope on the chyme flow in the small intestine. Srivastava [7] investigated a particular form of this problem under the approximation of purely axisymmetric and Newtonian fluid flow in a cylindrical annulus between the small intestine and the endoscope and restricted to case of a peristaltic rush wave as a pure single sine wave of long wavelength along the outer boundary of the annulus. The author calculated the pressure drop and the friction forces as functions of some parameters.

In the present study, we reformulate the forces acted by endoscope and the intestine on the flow of chyme in the presence or absence of the endoscope and calculate the pressure, pressure drop, leading order flow velocity and the forces due to the boundaries. Results are obtained with and without endoscope in the intestine and verify the results in particular operative regime which can be appropriate to the case flow of chyme during gastrointestinal endoscpy procedure in a human.

The present study is the first step of our plan to investigate chyme flow during gastrointestinal endoscopy. We are presently working on related problems but more realistic cases of non-axisymmetric flow, a non-Newtonian power-law fluid and superposed sine waves of different wavelengths, and the results of such studies will be reported elsewhere. This is consistent with our plan to understand the way intestinal infected disease can be diagnosed more effectively and thus improve the patient's health more quickly and with no possibility for complication due to lack of clear understanding of the factors that can contribute to the patient's illness. 


\section{Model Formulation}

We consider the problems of axisymmetric incompressible Newtonian fluid flow in a cylindrical annulus bounded at the outer boundary by the small intestine and at the inner boundary by the inserted endoscope. We assume that a sinusoidal peristaltic wave travels down the wall of the outer tube, which corresponds to the boundary of the small intestine (Figure 1). We describe the geometry of the outer tube surface as

$$
H(z, t)=r_{o}+A \sin [(2 \pi / \lambda)(z-c t)],
$$

where $r_{o}$ is the radius of the outer tube (intestine), $\mathrm{A}$ is the amplitude of the wave, $c$ is the wave speed, $t$ is the time variable and $z$ is the axial variable.

Due to the geometry of the physical problem, we consider cylindrical coordinate system. The governing equations for the continuity and the momentum of an axisymmetric Newtonian fluid flow in a fixed frame are given by White [8]

$$
\begin{gathered}
\partial(r u) / \partial r+\partial(r w) / \partial z=0 \\
\rho(\partial u / \partial t+u \partial u / \partial r+w \partial u / \partial z) \\
=-\partial p / \partial r+\mu\left(\nabla^{2} u-u / r^{2}\right), \nabla^{2} \\
=(1 / r) \partial / \partial r(r \partial / \partial r)+\partial^{2} / \partial z^{2} \\
\rho(\partial w / \partial t+u \partial w / \partial r+w \partial w / \partial z) \\
=-\partial p / \partial z+\mu\left(\nabla^{2} w\right),
\end{gathered}
$$

where $r$ is the radial variable, $z$ is the axial variable, $t$ is the time variable, $\rho$ is the fluid density, $\mu$ is the dynamic viscosity of the fluid, $u$ is the radial velocity, $\mathrm{w}$ is the axial velocity and $p$ is the pressure.

We now consider the governing system in the peristaltic wave frame of reference moving at speed $c$ of the wave, so that (2a)-(2c) become

$$
\partial(r u) / \partial r+\partial(r W) / \partial Z=0
$$

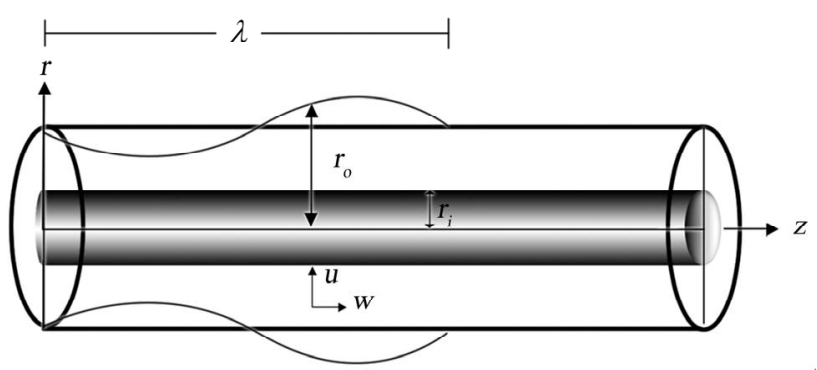

Figure 1. This is a schematic diagram for the physical system.

$$
\begin{aligned}
& \rho(\partial u / \partial t+u \partial u / \partial r+W \partial u / \partial Z) \\
& =-\partial p / \partial r+\mu\left(\nabla^{2} u-u / r^{2}\right) \\
& \rho(\partial W / \partial t+u \partial W / \partial r+W \partial W / \partial Z) \\
& =-\partial p / \partial Z+\mu\left(\nabla^{2} W\right),
\end{aligned}
$$

where

$$
Z=z-c t, W=w-c
$$

The boundary conditions in the moving frame of reference are

$$
\begin{aligned}
& u=0, W=-c \text { at } r=r_{i}, u=0 \\
& u=\partial H / \partial t=W(r=H, Z, t)(\partial H / \partial Z), \\
& W=-c \text { at } r=H
\end{aligned}
$$

where $r_{i}$ is the radius of the inner cylinder (endoscope).

Next, we non-dimensionalize the governing Equations (3a)-(3c) and the boundary conditions (4a)-(4b) using $r_{o}$, $\lambda, \lambda /\left(r_{o} c\right), c, \lambda / c,\left(\lambda c \mu / r_{o}\right)$ as scales for radial distance, axial distance, radial velocity, axial velocity, time and pressure, respectively. The non-dimensional form of the governing equations and the boundary conditions are then given below for simplicity using the same symbols that were used before for the independent variables, flow velocity and pressure

$$
\begin{gathered}
\partial(r u) / \partial r+\partial(r W) / \partial Z=0 \\
\varepsilon^{3} R_{e}(\partial u / \partial t+u \partial u / \partial r+W \partial u / \partial Z) \\
=-\partial p / \partial r+\varepsilon^{2}\left[(1 / r) \partial / \partial r(r \partial u / \partial r)-u / r^{2}+\varepsilon^{2} \partial^{2} u / \partial Z^{2}\right] \\
e R_{e}(\partial W / \partial t+u \partial W / \partial r+W \partial W / \partial Z) \\
=-\partial p / \partial Z+\left[(1 / r) \partial / \partial r(r \partial W / \partial r)+\varepsilon^{2} \partial^{2} W / \partial Z^{2}\right] \\
u=0, W=-1 \text { at } r=\delta \\
u=W(r=h, Z, t)(\partial H / \partial Z), \\
W=-1 \text { at } r=h \equiv 1+\Phi \sin (2 \pi Z)
\end{gathered}
$$

where $R=\rho c r_{o} / \mu$ is the Reynolds number, $\varepsilon=r_{o} / \lambda$ is ratio of the length scale in the radial to that in the axial direction, $\delta=r_{i} / r_{o}$ is the annulus aspect ratio, and $\Phi=$ $A / r_{o}$ is the non-dimensional amplitude of the wave.

We shall assume that the Reynolds number $R_{e}$ is sufficiently small and $\varepsilon$ is also a small parameter (wave length is large), so our leading order investigation neglects terms in (5b)-(5c) that contain such parameters. Such assumptions are consistent with the available observation by Lew et al. [9] that $R_{e}$ is very small and long wavelength approximation also exists in the small intestine. Under such assumptions, the Equation (5b) implies that the pressure $p$ is independent of the radial variation 
to the leading terms, and the Equation $(5 \mathrm{c})$ can be integrated twice with respect to $r$ leading to the result

$$
\begin{aligned}
& W= \\
& -1-0.25(\mathrm{~d} p / \mathrm{d} Z)\left[\delta^{2}-r^{2}+\left(\delta^{2}-h^{2}\right) \ln (r / \delta) / \ln (\delta / h)\right]
\end{aligned}
$$

after the necessary boundary conditions given in $(5 \mathrm{~d})-(5 \mathrm{e})$ are used.

Next, the dimensionless volume flow rate $q=\int_{S} W \mathrm{~d} S$, which is assumed to be given, in the wave frame along the axial direction is determined. Here $S$ is the crosssectional area of the annulus. We then find

$$
\begin{aligned}
& q= \\
& \pi\left(\delta^{2}-h^{2}\right)\left\{1+0.125(\mathrm{~d} p / \mathrm{d} Z)\left[\delta^{2}+h^{2}-\left(\delta^{2} h^{2}\right) / \ln (\delta / h)\right]\right\}
\end{aligned}
$$

Now, the relation between the $q$ in the wave frame and $q_{L}$ in the laboratory frame, which is the instantaneous one, is

$$
q=q_{L}-2 \pi \int_{\delta}^{h} r \mathrm{~d} r=q_{L}-\pi\left(h^{2}-\delta^{2}\right)
$$

The relation between the mean volume flow rates given by

$$
Q=\int_{0}^{1} q_{L} \mathrm{~d} Z
$$

in the laboratory frame and $q$ can then be found by taking the average of (8a), which yields the following equation:

$$
q=Q-\pi\left(1+\Phi^{2} / 2-\delta^{2}\right)
$$

The result (8c) is analog to that in the absence of endoscope [3]. Using (7), we can solve for $\mathrm{d} p / \mathrm{d} Z$ and then integrate over one wavelength in the axial direction and make use of (8c) to find the following expression for the pressure drop $\Delta p$ across one wavelength:

$$
\begin{aligned}
& \Delta p=\int_{0}^{1} G(Z) \mathrm{d} Z, G(Z) \equiv \\
& -\left\{8\left[Q / \pi+\left(h^{2}-1-\Phi_{2} / 2\right)\right] /\left[\delta^{4}-h^{4}-\left(\delta^{2}-h^{2}\right)^{2} / \ln (\delta / h)\right]\right\}
\end{aligned}
$$

Frictional forces $F_{i}$ and $F_{o}$ at the inner and outer boundaries, respectively, can be found from the integration of the shear stress around each tube and over one wavelength in the axial direction [10]. In analogy to the flow over a planner surface, we consider integral over one wavelength along the $Z$-direction of the dimensionless dominated term $\partial W / \partial y$ for the shear stress over the plate, where $y+r=r_{o}$. This leads to the expressions for these forces that are given below

$$
\begin{aligned}
& F_{\mathrm{i}}=\int_{0}^{1} \pi G(Z)\left\{\delta^{2}-\left(\delta^{2}-h^{2}\right) /[2 \ln (\delta / h)]\right\} \mathrm{d} Z \\
& F_{o}=\int_{0}^{1} \pi G(Z)\left\{h^{2}-\left(\delta^{2}-h^{2}\right) /[2 \ln (\delta / h)]\right\} \mathrm{d} Z
\end{aligned}
$$

It should be noted that the main results in the absence of the endoscope can be found by taking the limit $\delta \rightarrow 0$ in (9)-(10). This leads to

$$
\begin{aligned}
& \Delta p=\int_{0}^{1} G_{0}(Z) \mathrm{d} Z, G_{0}(Z) \equiv\left\{8\left[Q / \pi+\left(h^{2}-1-\Phi^{2} / 2\right)\right] / h^{4}\right\}, \\
& F_{i}=0, F_{o}=\int_{0}^{1} \pi h^{2} G_{0}(Z) \mathrm{d} Z
\end{aligned}
$$

which are similar to the results due to Srivastava and Saxena [11] for the single tube case after the relation (8c) is used to write $Q$ in terms of $q$.

\section{Results}

The main numerical results for the pressure drop and the frictional forces given by the analytical expressions (9)-(10) are determined using a computer code developed for the numerical integrations of these equations. The data were generated for the pressure drop $(\Delta p)$ and the frictional forces $\left(F_{i}\right.$ and $F_{o}$ ) for given values of the mean volume flow rate $(\mathrm{Q})$, amplitude of the wave $(\Phi)$ and the aspect ratio of the annulus $(\delta)$. Here a positive pressure drop $(\Delta p>0)$ means that pressure in the chyme flow decreases with increasing $Z$, while a negative pressure drop $(\Delta p<0)$ means that pressure in the chyme flow increases with $Z$. Due to the nature of the annulus flow and the way the expressions for $F_{i}$ and $F_{o}$ are determined in the previous section, it should be noted that a positive value of the inner friction force $\left(F_{i}>0\right)$ means this force is acted by the endoscope on the chyme flow, while $F_{i}<0$ means there such force acted by the chyme flow on the endoscope. However, in the case of the outer friction force, a negative value of $F_{o}\left(F_{o}<0\right)$ means that such force is acted by the intestine on the chyme flow, while a positive value of such force means that the chyme flow applies such force on the intestine.

Figures 2-4 present some typical results for the pressure drop, the inner friction force and the outer friction force versus the flow rate for different values of the amplitude of the wave and the aspect ratio of the annulus. It can be seen from the Figure 2 that the pressure drop is generally positive, provided the flow rate is not sufficiently small. Otherwise, the pressure drop is negative. In addition, for a given moderate value of $Q$, the pressure drop is positive and larger for larger values of aspect ratio and the wave amplitude. Thus, there is a pressure gradient force along the positive $Z$-direction, which acts 


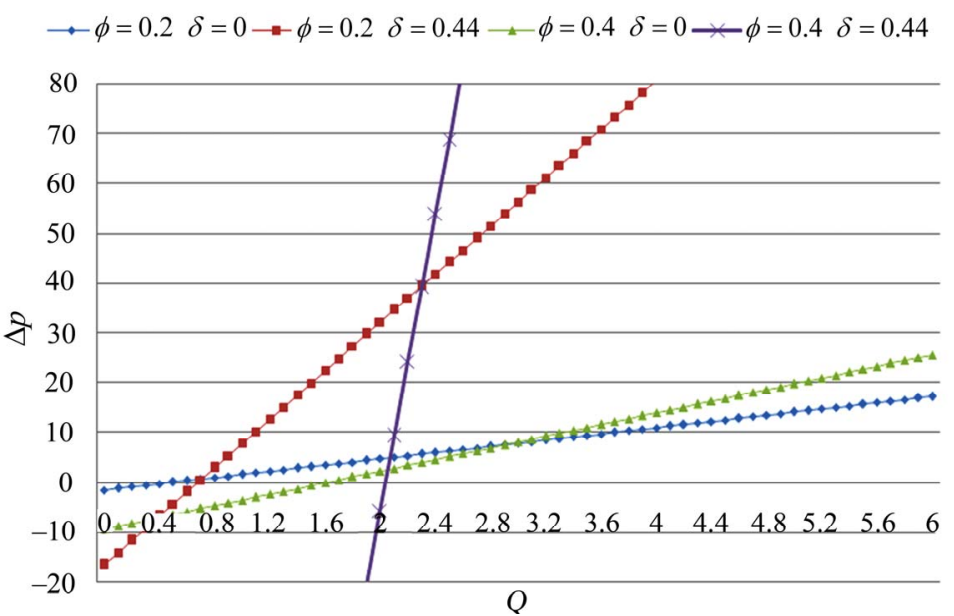

Figure 2.Pressure drop $\Delta p$ versus the flow rate $Q$ with the annulus aspect ratio $\delta=0,0.44$ and the wave amplitude $\Phi=0.2,0.4$.

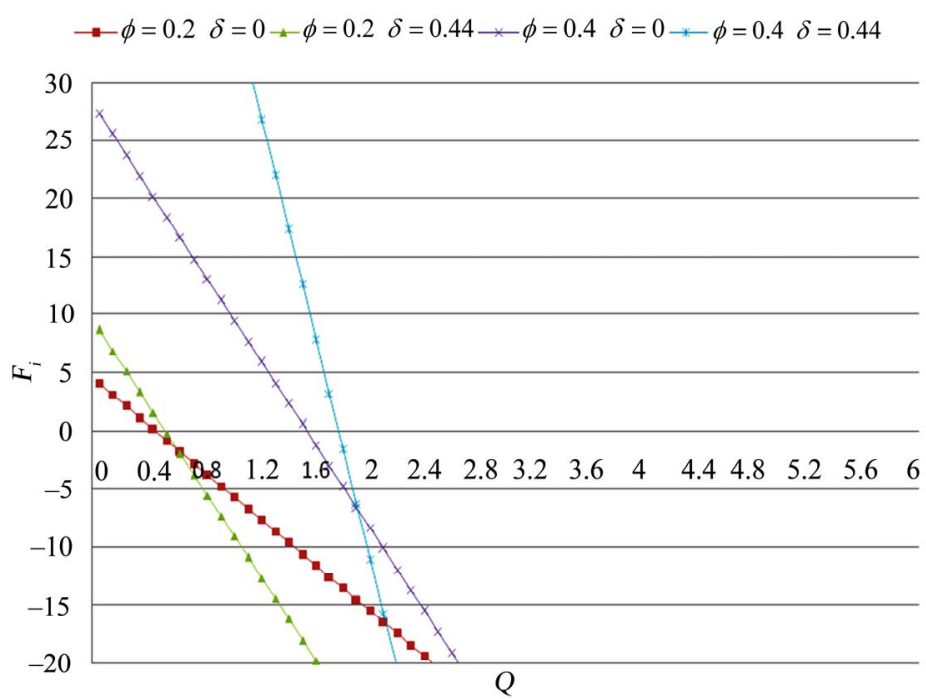

Figure 3. The inner friction force $F_{i}$ versus flow rate $Q$ with annulus aspect ratio $\delta=0.32,0.44$ and wave amplitude $\Phi=0.2$, 0.4 .

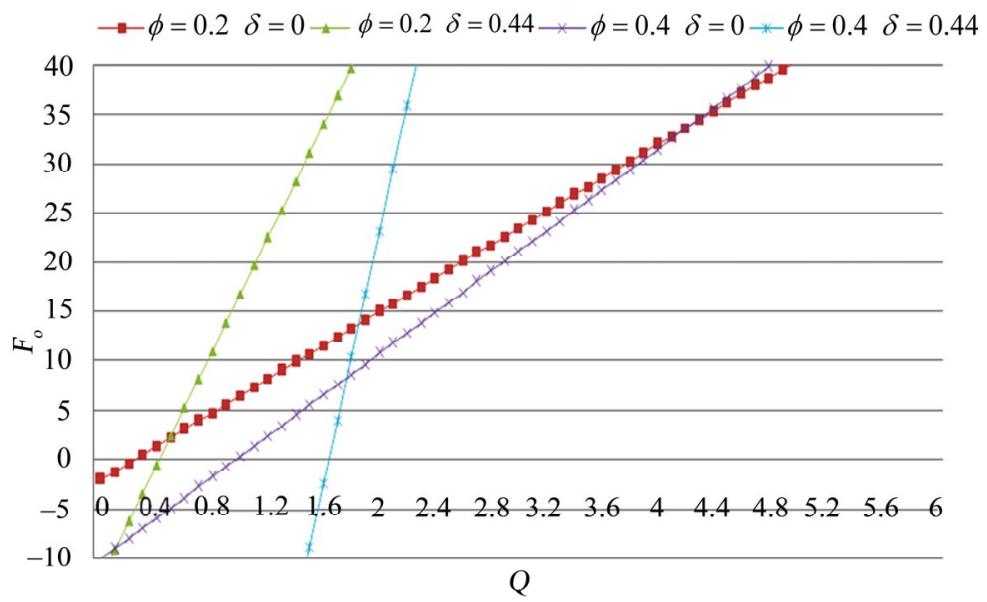

Figure 4. The outer friction force $F_{o}$ versus flow rate $Q$ with annulus aspect ratio $\delta=0.32,0.44$ and wave amplitude $\Phi=0.2$, 0.4 . 
on the flow, if the flow rate of the chyme flow is not too small, while such force takes opposite direction along negative $Z$-direction if the flow rate is too small. The magnitude of the pressure drop appears to increase significantly as either wave amplitude or the endoscope's cross sectional size increases. In analogy to that of flow over a surface, it is expected that negative pressure drop with sufficiently large magnitude can destabilize the chyme flow located between the intestine and the endoscope.

It can be seen from the Figure 3 that the inner friction force, $F_{i}$, is generally negative (i.e. the force acted by the chyme flow is on the endoscope), provided the flow rate is not sufficiently small. Otherwise, $F_{i}$ is positive. In the regimes where $F_{i}$ is either positive or negative, the magnitude of such force increases with either the wave amplitude or the endoscope's cross-sectional size. Thus, if the flow rate in the chyme flow is sufficiently small, then the force exerted by the endoscope on the chyme flow can have stronger effect if either the wave amplitude or the endoscope's cross-section are larger, while for sufficiently large values of the flow rate the force by the endoscope is replaced by that due to the chyme flow, which applies on the endoscope.

The results presented in the Figure 4 indicate that the outer friction force, $F_{o}$, is generally positive (i.e., the force is acted by the chyme flow on the intestine) provided $Q$ is not too small. Otherwise, $F_{o}$ becomes negative. For sufficiently large $Q, F_{o}$ is larger with larger value of either amplitude or the aspect ratio. For sufficiently small $Q$, where $F_{o}$ is negative, the magnitude of such forces increases with increasing either the amplitude or the aspect ratio. Thus, for sufficiently large flow rate of the chyme flow, the flow exerts a force on the intestine, and this force increases in its intensity for larger values of either the wave amplitude or the endo- scope's cross-sectional size. However, if the flow rate is sufficiently low, then there is a force acted by the intestine on the flow whose magnitude can increase with increasing either the wave amplitude or the endoscope's cress-sec- tion.

Figures 5-7 present some typical results for the pressure drop $\Delta p$, the inner friction force $F_{i}$ and the outer friction force $F_{o}$ versus the wave amplitude for different values of the flow rate $Q$ and the aspect ratio of the annulus $\delta$. It can be seen from the Figure 5 that for sufficiently small amplitude, the pressure drop is positive but is smaller for smaller aspect ratio and higher $Q$. However, for sufficiently larger amplitude, the pressure drop can be negative. Thus, the pressure gradient force in the chyme flow is in the positive direction of the $Z$-axis if the wave amplitude is sufficiently small, while such force has a direction in the negative $Z$-axis if the wave amplitude is sufficiently large, which can destabilize the flow system. Also such destabilizing effect can be intensified if the endoscope's cross-section is sufficiently large.

The results presented in the Figure 6 for the inner friction force indicate that for sufficiently small amplitude, the inner friction force is negative, and the magnitude of this force is larger for smaller $Q$ and larger aspect ratio. For sufficiently large amplitude, the inner friction force is positive and has larger magnitude for larger values of either $Q$ or $\delta$. Thus, the force acted by the flow on the endoscope intensifies if the flow rate is smaller and the endoscope's cross-section is larger. The force acted by the endoscope on the chyme flow is stronger if either volume flow or the endoscope's cross section is larger.

From the results presented in the Figure 7, we find that for sufficiently small amplitude, the outer friction force is positive which can have larger magnitude for either smaller flow rate $Q$ or larger annulus aspect ratio. For sufficiently large amplitude, the outer friction force is

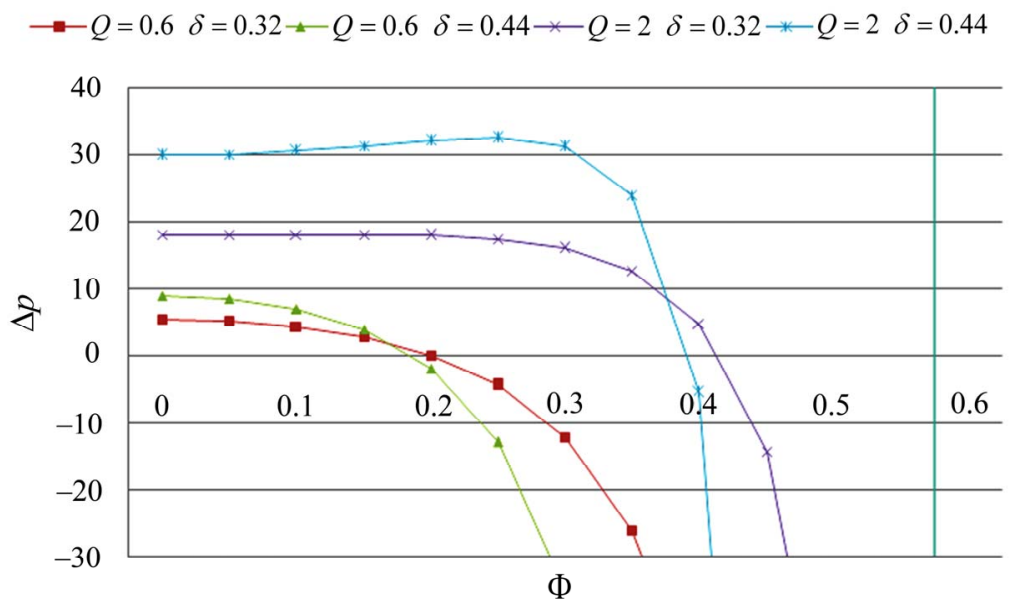

Figure 5. The pressure drop $\Delta p$ versus wave amplitude $\Phi$ for flow rate $Q=2,6$ and annulus aspect ratio $\delta=0.32,0.34$. 


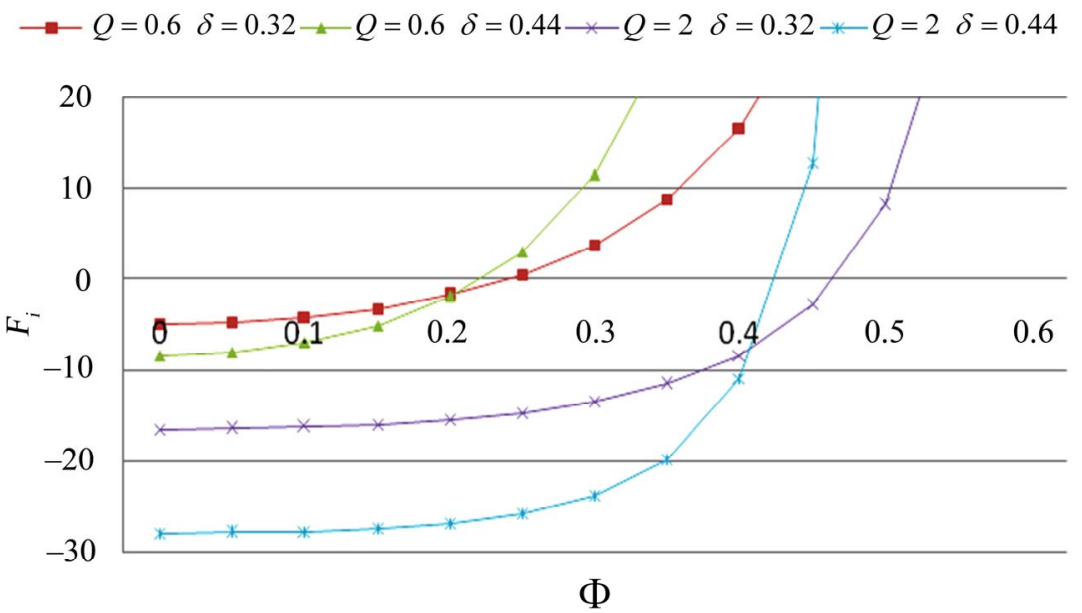

Figure 6. The inner friction force $F_{i}$ versus wave amplitude $\Phi$ for flow rate $Q=2,6$ and annulus aspect ratio $\delta=0.32,0.34$.

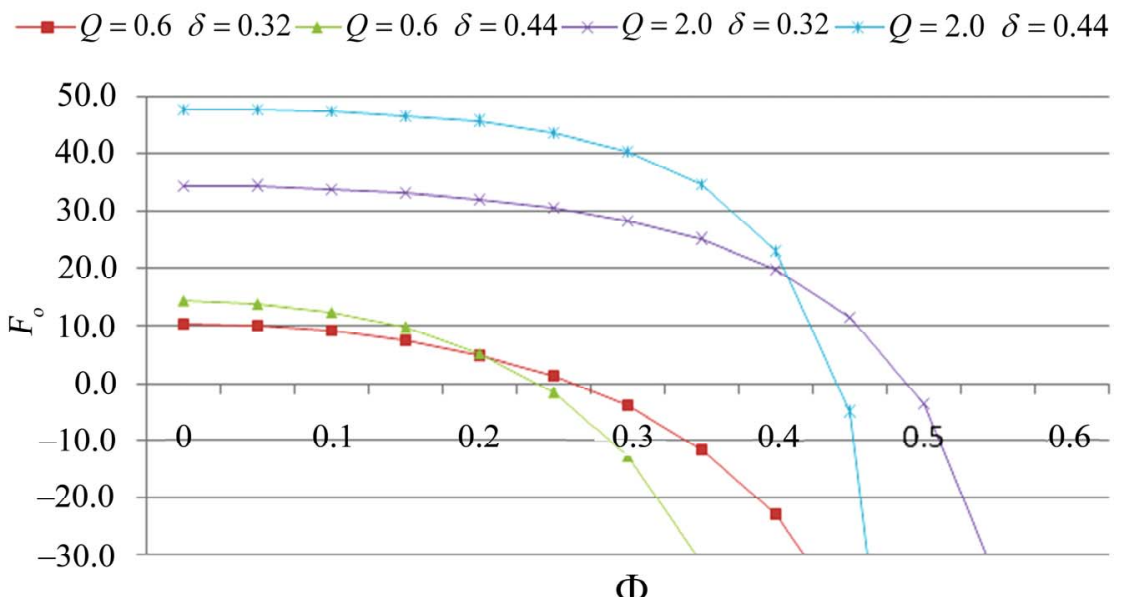

Figure 7. The outer friction force $F_{i}$ versus wave amplitude

for flow rate $Q=2,6$ and annulus aspect ratio $\delta=0.32,0.34$.

negative, and its magnitude is again larger for smaller $Q$ or larger $\delta$. Thus, the force exerted by the flow on the intestine is intensified either for larger endoscope's cross-section or for smaller flow rate. The intensification of the force acted on the flow by the intestine is similar to the case for force acted by the intestine of the chyme flow.

We have investigated leading order velocity flow and pressure versus axial variable for both annulus and tube flows for different flow rate, the annulus aspect ratio and the wavelength parameters. Figures 8-10 present some typical results for the pressure and velocity flow versus the axial variable. The parameters used for this analysis are the wave amplitude $\Phi=0.2$, annulus aspect ratio $\delta=$ $0.0,0.44$ and flow rate $Q=0.6,2.0$. Figure 8 presents results for pressure $(p)$. It can be seen from this figure that for the flow in the tube case, the pressure is lower for higher flow rate, which physically is reasonable, and $p$ mostly decreases with increasing $Z$ especially at higher flow rate. For the annulus flow, again $p$ is lower for high- er flow rate and decreases with increasing $Z$ for higher flow rate. Figure 9 presents some typical results for the leading order velocity $\mathrm{w}$ in the fixed frame versus $Z$. It can be seen from the Figure 9 that as anticipated the flow velocity is higher for higher flow rate. For the flow in the tube case, the flow direction is from left to the right, except in the case of low flow rate where the flow is to the left with relatively small magnitude in an intermediate zone in the axial direction. It is anticipated that in this zone flow circulate slightly. Similar results are apparent in the case of the flow in the annulus. Figure 10 presents some characteristic results for axial velocity $w$ versus aspect ratio $r$ for $r$ between annulus aspect ratio $\delta$ and outer tube surface $\mathrm{h}$ and at a axial distance $Z=0.5$. The parameter values are the same as those given for the Figures 8 and 9, respectively. It can be seen from the Figure 10, that $w>0$ and the value of the leading order velocity flow is higher for higher flow rate $Q$. The leading order velocity flow in the tube case is higher than that for $w$ in the annulus flow only if $r$ is sufficiently small. 


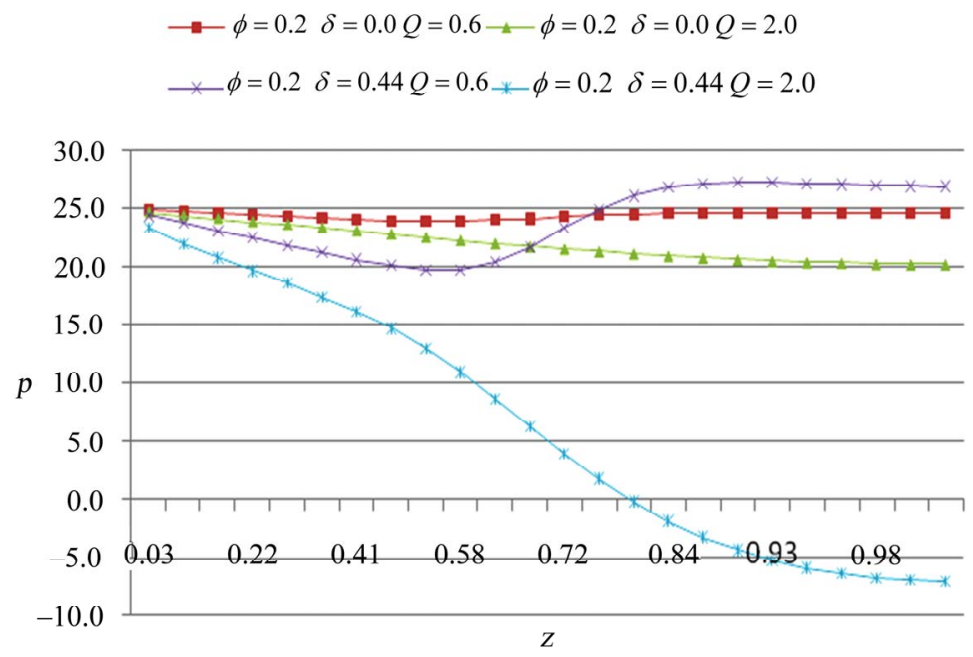

Figure 8. Pressure $p$ versus axial variable $Z$ for wave amplitude $\Phi=0.2$, annulus aspect ratio $\delta=0.0,0.44$ and flow rate $Q=$ 0.6, 2.0.

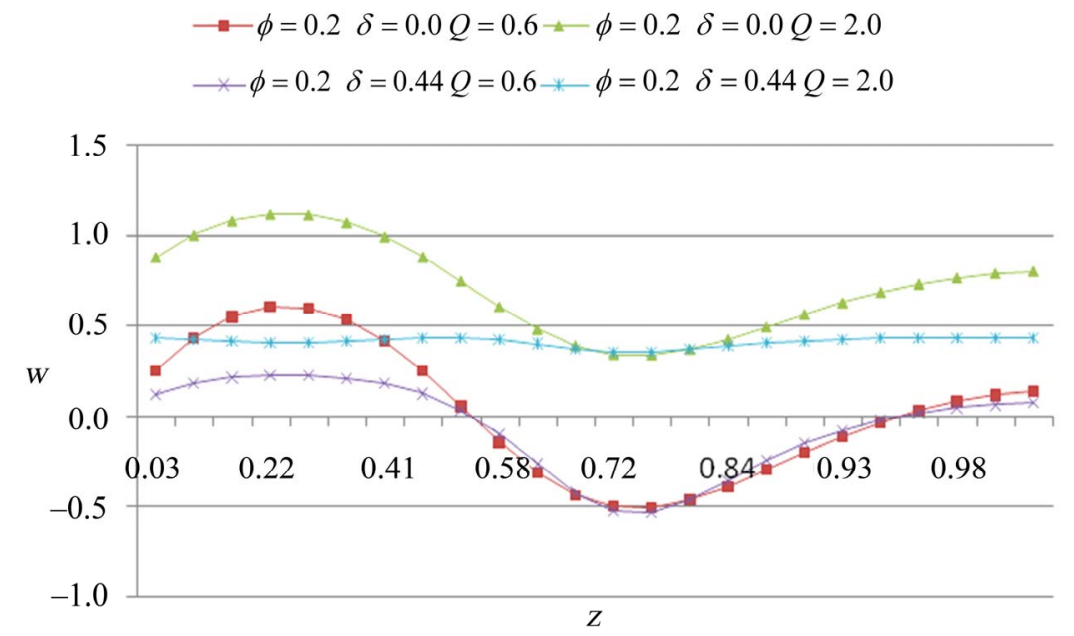

Figure 9. Axial velocity $w$ versus axial variable $Z$ at aspect ratio $r=0.5$ for wave amplitude $\Phi=0.2$, annulus aspect ratio $\delta=$ $0.0,0.44$ and flow rate $Q=0.6,2.0$.

$$
\begin{aligned}
& \longrightarrow \phi=0.2 \delta=0.0 Q=0.6 \multimap \phi=0.2 \delta=0.0 Q=2.0 \\
& \multimap \phi=0.2 \delta=0.44 Q=0.6 \longleftarrow \phi=0.2 \delta=0.44 Q=2.0
\end{aligned}
$$

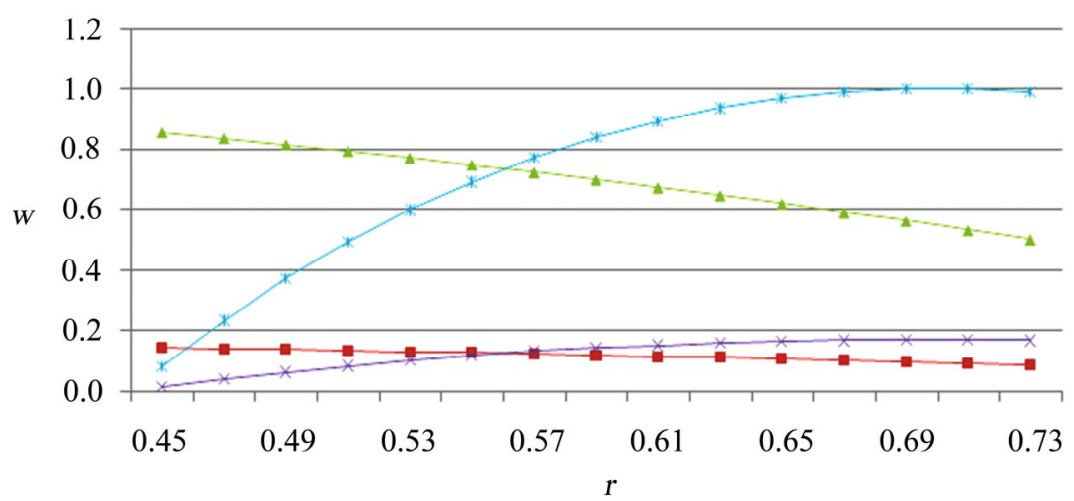

Figure 10. Axial velocity $w$ versus aspect ratio $r$ at $Z=0.5$ for wave amplitude $\Phi=0.2$, annulus aspect ratio $\delta=0.0,0.44$ and flow rate $Q=0.6,2.0$. 


\section{Conclusions \& Remarks}

We have carried out mathematical modeling and computation of a Newtonian incompressible fluid flow, under an axisymmetric condition, in a cylindrical annulus between the small intestine and an endoscope. We have developed the expressions for the pressure, pressure drop, leading order flow velocity in the flow and the integrated forces at the intestine and the endoscope over one wavelength of the peristaltic wave. We have computed the results for the pressure, flow velocity, pressure drop and forces for different flow rates, the amplitude of the wave, and endoscope cross-sectional size. We have seen that pressure decreases with increasing axial variables indicating that driving the pressure gradient force along the peristaltic wave and the pressure drop is generally positive. The magnitudes of the frictional forces are increased with increasing the wave amplitude or increasing the annulus aspect ratio or both. The leading order velocity flow is higher for higher flow rate, has higher amplitude in the absence of endoscope, decreases with increasing the radial variable $r$ in the absence of endoscope, while increases with $r$ in the presence of endoscope.

The purpose of the present investigation is to do modeling and computational study of the effect of an inserted endoscope on the flow of chyme in the small intestine using the simplest case by the consideration of axisymmetric flow of Newtonian fluid. Since boundary of the small intestine is not purely axisymmetric, a more realistic consideration for such flow system will be to consider non-axisymmetric flow system of either Newtonian fluid or more realistic power-law fluid [5,8]. Such study will be done in near future. Also as part of such future study, we will consider the system that models more realistically the flow of chyme and the peristaltic rush waves by assuming that such waves are composed of several sine waves with different wavelengths of moderately large size.

\section{Acknowledgements}

This research was supported by a grant from UTPA-URI.

\section{References}

[1] M. Y. Jaffrin and A. H. Shapiro, "Peristaltic Pumping," Annual Review of Fluid Mechanics, Vol. 3, 1971, pp. 13-36. doi:10.1146/annurev.fl.03.010171.000305

[2] T. W. Latham, "Fluid Motion in a Peristaltic Pump," Master's Thesis, Massachusetts Institute of Technology, Cambridge, 1966.

[3] A. H. Shapiro, M. Y. Jaffrin and S. L. Weinberg, "Peristaltic Pumping with Long Wavelength at Low Reynolds Number," Journal of Fluid Mechanics, Vol. 37, 1969, pp. 799-825. doi:10.1017/S0022112069000899

[4] L. M. Srivastava and V. P. Srivastava, "Peristaltic Transport of Blood. Casson Model II," Journal Biomechanics, Vol. 17, No. 11, 1984, pp. 821-829. doi:10.1016/0021-9290(84)90140-4

[5] L. M. Srivastava and V. P. Srivastava, "Peristaltic Transport of a non-Newtonian Fluid: Applications to Vas Deferens and Small Intestine," Annals Biomedical Engineering, Vol. 13, No. 2, 1985, pp. 137-153. doi:10.1007/BF02584235

[6] V. P. Srivastava, "Particle-Fluid Suspension Flow Induced by Peristaltic Waves in a Circular Cylindrical Tube," Bulletin of the Calcutta Mathematical Society, Vol. 5, No. 1, 2002, pp. 167-184.

[7] V. P. Srivastava, "Effects of an Inserted Endoscope on Chyme Movement in Small Intestine-A Theoretical Model," Applications and Applied Mathematics, Vol. 2, No. 2, 2007, pp. 79-91.

[8] F. M. White, "Viscous Fluid Flow," McGraw-Hill, Inc., New York, 1991.

[9] H. S. Lew, Y. C. Fung and C. B. Lowenstein, "Peristaltic Carrying and Mixing of Chyme in Small Intestine," Journal Biomechanics, Vol. 4, No. 4, 1971, pp. 297-315. doi:10.1016/0021-9290(71)90036-4

[10] L. D. Landau and E. M. Lifshitz, "Fluid Mechanics," 2nd Edition, Pergamum, London, 1987.

[11] V. P. Srivastava and M. Saxena, "A Two-Fluid Model of non-Newtonian Blood Flow Induced by Peristaltic Waves," Rheologica Acta, Vol. 34, No. 4, 1995, pp. 406-414. doi:10.1007/BF00367155 